\title{
A EDUCAÇÃO EM SAÚDE PARA PORTADORES DE DOENÇA CRÔNICA OFTÁLMICA: UM RELATO DE EXPERIÊNCIA
}

[Health education for ophtalmic chronic illness bearer: a report of experience]

\author{
Samanta Andrine Marschall Taube* \\ Mönica Cristina Espíndola Pimpäo Silva**
}

RESUMO: Este é o relato de uma experiência na especialidade de oftalmologia no cuidado ao cliente portador de doença crônica oftálmica e seus acompanhantes. Aenfermidade é responsável pelo acometimento de um número significativo de pessoas das mais variadas idades. Elaboramos um projeto de educação em saúde ao cliente portador de Retinopatia Diabética e Hipertensiva com os objetivos de disponibilizar orientações para elevar o conhecimento do cliente sobre sua enfermidade e incentivar questionamentos, proporcionar conforto durante os exames e contribuir à reflexão sobre condições e hábitos de vida do cliente. Após a aplicação deste projeto, percebemos a contribuição das orientações à conscientização da necessidade do tratamento da enfermidade crônica oftálmica e à maior aderência ao mesmo, bem como à qualidade do cuidado prestado, tranqüilidade manifestada pelo cliente durante a realização dos exames e sua participação durante a realização da Educação em Saúde.

PALAVRAS-CHAVE: Educação em saúde, enfermagem, doença crônica.

\section{A INTRODUÇÃO DO TEMA}

As enfermidades crônicas demandam um olhar constante dos profissionais de saúde, pois

\footnotetext{
*Enfermeira. Especialista em controle de infecção hospitalar pela São Camilo/Sul e em centro cirúrgico e central de material e esterilização pela Unioeste/PR. Atuante na especialidade de Oftalmologia.

${ }^{* *}$ Enfermeira. Especialista em ClH pelas Faculdades São Camilo - SP.

Atuante na especialidade de Oftalmologia
}

acometem grande parte da população das mais variadas faixas etárias as quais são, inevitavelmente, destinadas a conviver com mudanças significativas em seu cotidiano de vida. Smeltzer e Bare (1998) referem-se à doença crônica como um problema de saúde que necessita de uma administração dos aprendizados e da vivência com sinais, sintomas e características que se farão presentes em longo prazo além de, ao mesmo tempo, haver necessidade de aprender a viver com alterações de identidade que a doença fará existir. Tais problemas de saúde afetam as pessoas durante toda a vida, podendo ocorrer em todas faixas etárias sendo, entretanto, mais comum em um determinado grupo (população adulta e idosa) do que em outro. Conforme os autores acima citados, as enfermidades crônicas tendem a aumentar freqüentemente à medida que $a$ idade avança, sendo que muitos idosos as desenvolvem de forma múltipla. Além disso, acometem todos os gêneros, níveis sócio-econômicos, étnicos, culturais e raciais, porém são mais freqüentes em indivíduos pertencentes a grupos sócioeconômicos baixos devido ao pouco acesso aos cuidados de saúde, nutrição precária e hábitos de vida pouco saudáveis.

Sobre o impacto das doenças crônicas nas atividades ou no estilo de vida dos portadores e na sua dependência ou não de cuidados, Smeltzer e Bare (1998) apontam que pode ser nulo, baixo ou elevado, o que vai depender do grau de acometimento da enfermidade e de outros fatores pessoais associados. Este pensamento pode ser observado no trecho a seguir: 


\begin{abstract}
"As pessoas com problemas de cuidados de saúde crônicos podem funcionar independentemente e levar uma vida plena apenas com o mínimo de distúrbios; outras podem requerer uma monitorização freqüente e controlada ou uma permanente estada em um local de cuidados de problemas de longa duração" (SMELTZER \& BARE: 1998, p.275).
\end{abstract}

O grau de dependência encontra-se relacionado ao tipo de enfermidade crônica existente e ao potencial de acometimento físico das mesmas, sendo que as doenças sistêmicas crônicas que mais acometem a população adulta e que podem causar alterações no fundo do olho são a Hipertensão Arterial Sistêmica (HAS) e o Diabetes Mellitus (DM)

Assim, Oliveira e Kara-José (2000) comentam que dentre as enfermidades oculares relacionadas à presença de doença crônica, freqüentes nos clientes da especialidade de oftalmologia, encontram-se as presentes no fundo do olho ou no segmento posterior: retinopatias hipertensiva e diabética, sendo esta última a principal causa de cegueira na idade adulta, atingindo cerca de $12 \%$ de todos os cegos nos países ocidentais. "A cegueira da retinopatia diabética é, no momento, o maior problema de saúde publica ocular" (OLIVEIRA \& KARAJOSÉ:2000, p.133).

Portanto, pensamos ser importante comentar brevemente aspectos que envolvem as retinopatias, como a sintomatologia desta doença crônica oftalmológica. Sobre os sinais e sintomas da retinopatia hipertensiva, Oliveira e Kara-José (2000) citam que inicialmente podem ser observadas as seguintes alterações: estreitamento arteriolar, exsudatos duros e micro-hemorragias, exsudatos algodonosos (indicadores de morte celular focal) e edema de papila em casos mais graves. Já na retinopatia diabética, segundo os mesmos autores, ocorre uma divisão da doença em dois tipos: não proliferativa e proliferativa. $\mathrm{Na}$ primeira há presença de micro-hemorragias, microaneurismas, exsudatos duros e algodonosos, tortuosidade venosa e anormalidades microvasculares intra-retinianas com edema macular freqüente. Já na segunda, que ocorre em $5 \%$ dos casos, anormalidades vasculares aparecem no interior da cavidade vítrea e superfície da retina, sendo que a perda da visão pode ser severa provocando um descolamento da retina.

A orientação e o conhecimento dos aspectos que envolvem a doença por parte dos portadores, citados anteriormente, são de extrema importância, pois segundo Oliveira e Kara-José (2000, p.133)

\begin{abstract}
"o acompanhamento clínico e oftalmológico periódico, visando detectar a retinopatia em estágios iniciais e o precoce tratamento ocular, retarda e, muitas vezes evita, a perda visual."
\end{abstract}

As pessoas com Diabetes Mellitus e/ou Hipertensão Arterial Sistêmica devem ter conhecimento do risco de desenvolver retinopatia e, para tanto, devem ser orientadas sobre os possíveis sinais e sintomas da doença, sobre os cuidados necessários para evitar o seu desenvolvimento e, em caso de acometimento ocular, sobre a importância de realizar acompanhamento oftalmológico regularmente para controle da patologia ocular, uma vez que estas informações poderão prevenir o agravamento da doença. "O diagnóstico e o tratamento precoce por um especialista em retina pode reduzir enormemente o risco de grave perda visual" (SMELTZER \& BARE: 1998, p.1388).

Neste contexto, o enfermeiro aparece na atuação prática frente aos portadores de doenças crônicas com o intuito de buscar variadas alternativas que possam propor a melhoria e/ou mudanças nos hábitos e nas condições de vida de seus portadores, com o objetivo de facilitar o aprendizado e a convivência com a doença crônica, sendo as orientações, por meio da educação em saúde, um instrumento que auxilia alterações graduais e significativas ao seu estilo de vida e à prevenção de complicações da doença. Neste sentido, Smeltzer \& Bare (1998) comentam que mudanças no estilo de vida são almejáveis para o controle de sinais e sintomas e para prevenir possíveis complicações.

O enfermeiro que trabalha com a especialidade de oftalmologia atua de várias formas no cuidado ao cliente, mediante embasamento teórico-científico e provido de instrumentos que direcionam este cuidado articulando assim, a ciência e a arte da enfermagem. Entendemos que as orientações, através da educação em saúde ${ }^{1}$, fazem parte do trabalho do enfermeiro independente

\footnotetext{
'Neste trabalho ao ler-se Educação em Saúde entenda-se Atividade
} educativa em grupo. 
da área ou especialidade em que atua, sendo um importante e necessário instrumento ao cuidado. Em encontro com esta afirmação citamos PEREIRA \& LIMA (2002, p.143) quando dizem que:

"A atividade educativa em grupo é um importante instrumento de trabalho do enfermeiro, no enfrentamento de uma questão de saúde de grande prevalência (...), pois permite prestar assistência específica a várias pessoas com necessidades semelhantes, aproveitando melhor o tempo e a riqueza de troca de experiências."

A prática de cuidado ao cliente oftalmológico revela a presença de angústia, desorientação e um acúmulo constante de dúvidas por parte do cliente, principalmente sobre o contexto que envolve o seu tratamento, realizado durante 0 atendimento médico e de enfermagem no ambulatório da especialidade. É evidente que os clientes, de um modo geral, mostram-se ansiosos sobre as questões que norteiam seu diagnóstico e tratamento e que, na maioria das vezes, deixam a instituição de saúde com mais dúvidas do que quando nela adentraram. Motivo este que se deve, dentre outros, ao curto tempo de permanência no consultório médico, a deficiente orientação que lhe é prestada por parte dos profissionais de saúde ou até mesmo, pela inibição do próprio cliente em levantar questionamentos.

A partir destas observações percebemos que os objetivos à implantação do projeto foram coerentes com as necessidades dos clientes da Instituição, e que muito desta ansiedade, sentida e transmitida por eles, poderia ser sanada e/ou minimizada por meio de um programa de educação em saúde. Smeltzer \& Bare (1998) afirmam que o medo e a ansiedade podem ser reduzidos com a partilha de informações acerca dos resultados de exames com o cliente na explicação do diagnóstico e do plano de tratamento, de modo a promover a sua participação. Estas atitudes proporcionarão sensação de controle e autonomia e auxiliarão a reduzir o medo e a ansiedade.

\footnotetext{
"A ansiedade freqüentemente sofrida pelo paciente oftalmológico exige consideração quanto à sua condição física. A dependência que uma pessoa tem da sua vista é enfatizada quando se enfrenta uma perda temporária, ou possivelmente, permanente desse sentido vital. O aborrecimento, o medo e a depressão são reações comuns; a tensão, o
}

ressentimento, a irritação e a rejeição podem também ocorrer" (SMELTZER \& BARE, 1998:1394).

Frente ao exposto, os objetivos foram os de disponibilizar orientações com intuito de elevar o conhecimento do cliente sobre sua enfermidade e a necessidade de tratamento efetivo, incentivando o questionamento direto aos profissionais de saúde sobre questões que norteiam a enfermidade apresentada, proporcionar maior conforto ao cliente durante a realização dos exames, auxiliar o processo de realização dos exames, tornar a sala de espera um ambiente produtivo e de educação em saúde ao invés de penoso e angustiante, além de contribuir para reflexões sobre as condições e hábitos de vida do cliente portador de uma doença crônica em seu domicílio.

O objetivo deste artigo é relatar a experiência vivenciada no cuidado de enfermagem ao cliente portador de doença crônica oftálmica e seus acompanhantes, relacionado aos procedimentos diagnósticos e terapêuticos necessários ao controle da enfermidade.

\section{O PROJETO DE EDUCAÇÃO EM SAÚDE NA SALA DE ESPERA DO SETOR DE EXAMES OFTALMOLÓGICOS AO PORTADOR DE RETINOPATIA: A NOSSA EXPERIÊNCIA}

O projeto de educação em saúde envolveu a descrição dos objetivos, justificativas à implantação, metodologia de ação e levantamento de recursos necessários à sua realização. Depois de elaborado, foi apresentado à administração da Instituição, a qual aprovou a sua implantação.

A etapa seguinte envolveu uma primeira aplicação do projeto por uma das enfermeiras, como um piloto (teste), por meio da realização da Educação em Saúde aos portadores de retinopatia que foram encaminhados à Instituição para realizar exames de diagnose e terapêutica. Na ocasião, contamos com 21 participantes, dentre eles clientes e seus acompanhantes, que foram encaminhados à sala de espera de exames da Instituição. A coleta de assinaturas dos participantes foi realizada para registrar a participação.

Cabe aqui ressaltar que, a maioria dos clientes portadores de retinopatias, participantes das atividades de educação em saúde, apresentou 
certo grau de acometimento visual, o que explicou a necessidade de acompanhantes durante a realização do tratamento oftalmológico.

Durante a implementação, ficou constatada a necessidade de elaborar um instrumento que conduzisse as exposições educativas das enfermeiras, uma espécie de roteiro com intenção de ser estabelecido um padrão mínimo a ser seguido, com vista ao tempo reduzido à sua realização e à necessidade de exposição de questões fundamentais envolvendo as retinopatias, seu tratamento e orientações de enfermagem. $O$ instrumento possui esta seqüência: apresentação pessoal, objetivos, abordagem de questões relacionadas às doenças sistêmicas (Hipertensão Arterial Sistêmica e Diabetes Mellitus), relacionadas ao surgimento de retinopatias tais como conceito e/ou definição das patologias e sua influência ao desencadeamento de retinopatias, aspectos sobre a realização dos exames de diagnose e terapêutica como a angiofluoresceinografia e a aplicação de laser de argônio.

Sobre os exames de diagnose e terapêutica das retinopatias, Oliveira e Kara-José (2000) apontam que a angiofluoresceinografia consiste em uma técnica de injeção de contraste (fluoresceína sódica) intravenosa seguida da avaliação do trajeto deste contraste nos vasos da coróide e retina, sendo um exame importante no acompanhamento de retinopatias diabética e hipertensiva, alterações da mácula, tumores oculares e outros. Já a aplicação de laser de argônio, como referem os mesmos autores, tem por objetivo a fotocoagulação, através da propagação paralela de uma luz pura a qual é dirigida para o tecido, absorvida pelo pigmento presente no mesmo (melanina ou hemoglobina) e transformada em energia térmica, capaz de elevar a temperatura e coagular o tecido, da qual resulta uma cicatriz. Ainda relatam que:

"O laser de argônio (...) é utilizado para o tratamento de retinopatia diabética, descolamento de retina, destruição de neovasos retinianos, lesões degenerativas da retina, que predispõem ao descolamento, para fotocoagulação de vasos anormais na íris e no tratamento de glaucoma, através de trabeculoplastia" (OLIVEIRA \& KARAJOSÉ: 2000, p.282).
Também foram fornecidas orientações durante a realização dos exames, dando-se ênfase à importância da adesão do cliente ao tratamento oftalmológico da retinopatia e também aos cuidados domiciliares necessários para o controle da doença crônica, bem como para mudanças no estilo e hábitos de vida por meio da reflexão e conscientização de sua importância de realização.

$\mathrm{O}$ instrumento elaborado foi respaldado cientificamente segundo Smeltzer \& Bare (1998) e Oliveira \& Kara-José (2000), ao abordarem as atividades educativas necessárias ao portador de doença crônica, onde se destacam orientações sobre o conhecimento e o incentivo do cliente no cumprimento da dieta alimentar e o uso adequado das medicações para controlar os níveis de glicemia e hipertensão. Outra orientação pertinente esta na realização de exames oftalmológicos regulares periódicos, os quais podem detectar sinais de retinopatias muito tempo antes de os sintomas se tornarem evidentes ao portador. Além disso, os autores enfatizam a necessidade de iniciar medidas numa tentativa de controlar, assim como prevenir, a possível progressão da deterioração ocular provocada pela doença crônica.

\section{DINÂMICA DAS ATIVIDADES DE EDUCAÇÃO EM SAÚDE}

A sala de espera do setor de exames foi o local escolhido primeiramente por ser um ambiente amplo, com disponibilidade de cadeiras, separado de outros setores da Instituição e também por ser o local de destino dos clientes portadores de retinopatias encaminhados à realização de exames e/ou tratamento de tal patologia.

O período, o horário e o tempo dispostos à educação em saúde foram definidos e baseados na programação das atividades da Instituição, especificamente nos dias de realização dos exames pela mesma: manhãs das terças e quintasfeiras. O horário escolhido para as atividades educativas foi o das 8:30, pelo fato dos clientes já estarem preparados (já passaram pela recepção, atividades burocráticas e preparo pré-exame) e aguardando a realização dos exames na sala de espera. A duração da educação em saúde foi determinada em 20 (vinte) minutos, devido as enfermeiras concordarem que este seria o tempo adequado e suficiente para a exposição das 
que surgiam durante a exposição. Além disso, existia a necessidade de iniciar a realização dos exames de diagnose e terapêutica pelos médicos oftalmologistas, o que determinou a adequação do tempo.

As orientações de educação em saúde apresentaram periodicidade semanal e/ou sempre que se fizeram necessárias. Isto se deu ao fato da Instituição apresentar dias da semana específicos à realização de exames oftalmológicos, nos quais os clientes portadores de retinopatias eram encaminhados à mesma para a realização de exames oftalmológicos de diagnose (angiofluoresceinografia) e de terapêutica (laser de argônio).

No que diz respeito à forma de abordar e realizar as orientações foram escolhidos a exposição e o diálogo, sendo que este último foi por nós considerado uma rica fonte de troca de informações e questionamentos por parte do grupo. Para que esta abordagem fosse possível, utilizamos como recursos audiovisuais cartazes (com figuras da anatomia ocular) e uma maquete de um olho humano.

Fica importante mencionar que durante os encontros de educação em saúde questionamentos surgiram, com um interesse maior voltado para a possibilidade de um agravamento da alteração ocular e cegueira, à existência de cuidados domiciliares que pudessem auxiliar e/ou melhorar a evolução do quadro e à finalidade das sessões de aplicação de Laser. Tais dúvidas foram sanadas pelas enfermeiras no decorrer do diálogo, com base nas referências bibliográficas encontradas sobre o tema que, inclusive, serviram como base para a elaboração do projeto. O surgimento deste fato contribuiu para a ênfase dada pelas enfermeiras nos encontros posteriores sobre estas questões, o que proporcionou melhor qualidade na exposição do assunto.

Por fim houve a solicitação, após o término de cada encontro, que os participantes assinassem um livro de presença no programa educativo. $O$ livro foi aberto para que as assinaturas pudessem registrar a presença e participação no recebimento de orientações na sala de espera.

\section{ALGUMAS CONSIDERAÇÕES}

Considerando a doença crônica uma enfermidade evidente e crescente na população em geral, as retinopatias como principais causas de cegueira nos pacientes portadores de doenças crônicas como hipertensão e diabetes e a necessidade de intervenções efetivas que contribuam nas condições de vida dessa população, houve a necessidade de elaborar este programa de orientação.

Os clientes oftalmológicos apresentam considerável déficit de conhecimento sobre os aspectos que envolvem sua enfermidade e sobre o tratamento necessário. É papel dos profissionais de saúde, especificamente dos enfermeiros, oferecerem orientações sobre as necessidades de mudanças e participação nesta mudança por parte dos clientes e familiares. Temos a Educação em Saúde como um instrumento de trabalho da enfermagem voltado ao cuidado do cliente, a qual deve ser utilizada de modo à abranger o cotidiano da prática profissional, para que a efetividade e a adesão ao tratamento, pelo cliente, sejam encorajadas e entendidas como necessárias à obtenção de mínimas condições de bem-estar e à redução da progressividade da doença oftalmológica, causada pela doença crônica sistêmica.

Um fator que contribuiu para um bom desempenho na Educação em Saúde foi a utilização de recursos visuais (como a maquete de um olho humano) para explanar a relação da doença crônica com a alteração provocada por ela no fundo do olho (retinopatia), os quais foram de suma importância para o entendimento dos participantes, ficando comprovada a sua utilidade nas orientações fornecidas durante a educação em saúde. Vale ressaltar que alguns participantes demonstraram interesse em manipular a maquete, revelando a curiosidade sobre a anatomia do olho humano e as complicações que poderiam ocorrer neste órgão devido a patologia crônica que apresentavam.

Tivemos, com a implementação deste trabalho educativo e informativo, os objetivos de intervir no cotidiano das pessoas portadoras de retinopatias, por meio da disponibilização de 
orientações, do incentivo ao questionamento dos clientes e acompanhantes, o que tornou a sala de espera um ambiente produtivo e instigante e, também, de auxiliar a realização dos exames de diagnose e terapêutica e com isso, melhorar as condições e hábitos de vida desta população.

Percebemos que, ao final da análise das ações educativas realizadas, os objetivos propostos foram alcançados na contribuição ao crescimento dos participantes, clientes e acompanhantes, no que se refere ao conhecimento da enfermidade ocular, ao tratamento a ela dispensado e a necessidade de adesão ao tratamento e aos cuidados domiciliares pertinentes à doença crônica, também na redução de sentimentos como o medo e a ansiedade acerca do tratamento realizado na Instituição de Saúde e na possibilidade de auxiliar o cliente durante a realização dos seus exames de diagnose e terapêutica. Outra observação foi a do crescimento das enfermeiras, advindo da elaboração do projeto e da contribuição dos clientes e acompanhantes por meio dos questionamentos que surgiram durante as atividades educativas, o que facilitou o reconhecimento das necessidades particulares do grupo o qual nos fez refletir sobre a importância das orientações específicas e do diálogo, além da percepção do quanto tais atividades podem contribuir à melhora das condições de vida e convivência do cliente e seus acompanhantes com a doença crônica oftalmológica.

No decorrer da educação em saúde percebemos um constante e crescente interesse vindo dos participantes (pacientes e acompanhantes) e, especialmente, dos acompanhantes que cresceram, também, em presença durante as orientações. Aproveitamos esta presença para realizar orientações voltadas às adequações necessárias no domicílio (mudanças de hábitos e estilo de vida) e da sua importância no auxílio e encorajamento à adesão do tratamento pelo portador da doença crônica oftalmológica.

O alcance dos objetivos propostos só foi possível devido ao interesse do grupo em participar do trabalho desenvolvido e ao planejamento e conhecimento buscados pelas enfermeiras à realização deste projeto, o que possibilitou a construção de uma nova forma de atuação no cuidado ao cliente oftalmológico, valendo-se de disposição, criatividade e conhecimento por parte das enfermeiras, na medida que as necessidades de orientações foram surgindo durante o cotidiano da prática profissional.

Ao nosso olhar, ser enfermeira em qualquer especialidade significa articular o saber e o fazer por meio de instrumentos, de conhecimentos científicos e da observação das necessidades que norteiam a sua práxis.

Ao elaborar e implementar este projeto, propomos uma maneira de fazer a enfermagem no contexto da especialidade de oftalmologia, de modo a instigar a busca por alternativas de cuidado que atendam as reais necessidades dos clientes e familiares/acompanhantes.

Sugerimos que outras instituições realizem esta alternativa de cuidado de enfermagem ao cliente crônico oftalmológico, e que outras propostas sejam lançadas com o objetivo de aprimorar as pesquisas e as intervenções na prática profissional do enfermeiro da especialidade de oftalmologia.

ABSTRACT: This is a report of the practical experience in specialty of ophthalmic in the care bearer chronic illness ophthalmic client and his companion. The illness is responsible for the attack of a significant number people of more ages varied. We prepared a project of health education for bearer client of diabetic and hipertensiv rethinophatic with the objective of availably directions for to lift up the client know ledge about his illness, was incentive questions, proportioning comfort during the examinations and contributing for conditions and customs client's life. After the application of this project it was noticed the directions contributions executioner for conscience of treatment necessity of illness ophthalmic chronic, for greater adherence for it, as well as quality of given care, for client tranquility demonstrated for the client during the examinations execution and his participation during the execution of heath education.

KEY WORDS: Heath education, nursing, chronic illness.

\section{REFERÊNCIAS}

BITTER JÚNIOR, A; MATHEUS, M.C.C. Comunicação In: CIANCIARULLO, T. I. Instrumentos básicos para o cuidar um desafio para a qualidade da assistência. São Paulo: 
Atheneu, 1996.

BONANOMI, M. T. O que é laser e mecanismo de sua ação - In: I Congresso de Auxiliar de Oftalmologia, 1999, São Paulo. Anais. São Paulo, 1999.

OLIVEIRA R.C.S. \& KARA-JOSÉ, N. Auxiliar de oftalmologia, São Paulo:Roca, 2000.

PEREIRA, G.A.; LIMA, M.A.D.S. Relato de experiência com grupo na assistência de enfermagem a diabéticos. Rev. Gaúcha Enferm., Porto Alegre, v.23, n.1, p. 142-157, jul., 2002.

REMEN, N. Paciente como ser humano, São Paulo: Atheneu, 1992.

RUIPÉREZ, I. \& LLORENTE, P.. Geriatria. Rio de Janeiro: McGraw Hill, 2000

SMELTZER, S. C.; BARE, B.G. Brunner \& Suddarth tratado de enfermagem médicocirúrgica. $8 \mathrm{Ed}$. Rio de Janeiro: Guanabara Koogan, 1998.

ENDEREÇO DAS AUTORAS: Rua Walenty Golas, 370 - ap.304-B Campo Comprido 81200-520 Curitiba/PR samarschall@bol.com.br 